\title{
Design and Simulation of MEMS Based Gyroscope
}

\author{
Sujit Kumar, B. Hemalatha \\ Department of Instrumentation and Control EngineeringSRM University, KattankulathurChennai, Tamilnadu- \\ 603203, India
}

\begin{abstract}
This paper represents a MEMS based gyroscope to measure orientation. Silicon-micro-machined gyro is fabricated on the basis of resonators and they use vibrating mechanical element to sense rotation. The vibratory measurement can be done by measuring the change in capacitance with respect to the voltage or force applied in particular axis. Here MEMS based gyroscope has been designed from Lame mode resonator which is a square plate. The additional benefits by using the gyroscope are lower sensor cost, power consumption, more robustness, higher shock resistance. In this paper, also sensitivity of the geometry and change of capacitance has been shown.

Index Terms-Coriolis Force, Gyroscope, MEMS.
\end{abstract}

\section{INTRODUCTION}

Equipment for sensing, which includes the cameras, navigational instruments, electronic imaging devices etc., are frequently carried by moving vehicle for different purposes and operated in the vehicle, such as airplane which undergoes rotational motion about its center of rotation. MEMS based gyroscope is an inertial sensor which provides the angular orientation of such sensing equipment. Gyroscope has two mode- driving and sensing mode.Proposed design of gyroscope is in 3-axis whose working principle is based on the transfer of energy between driving mode \& sensing mode of gyroscope which is caused by Coriolis acceleration. Whenever gyroscope will be subjectedto rotate in respective rotating axis then sensing mode will sense that rotation in terms of change in capacitance. A significant costdriver in traditional stabilization systems is thegyroscope. With the advanced technology of MEMS, MEMS based gyroscope has been applied to automotive application, robotic \& military application for different purpose. MEMS gyroscopes have a wide application spectrum in the automotive and consumer electronics markets [1]. This is due to their reduced costs, size and integration capability. Vibrating micro-machined gyroscopes that utilize vibrating elements to induce and detect coriolis force have been effectively implemented and demonstrated in various micromachining-based batch fabrication processes.

\section{Working PRINCIPLE OF A Gyroscope}

A Gyroscope is a sensor that measures the rate of rotation of an object. Since gyroscope has two modes for actuating and sensing. Vibrating gyroscopes must be driven at resonance in order to function as angular rate sensors. This direction will be referred as the drive direction. When the device is rotated along the rotation axis, a coriolis force is induced in the sense direction which will be orthogonal to both axis. The force will excite the device in sense direction into resonance mode. The sense direction is orthogonal to both the drive direction and the rotation axis. The sense and drive direction, can be viewed as a mass-spring damper system. Hence, a gyroscope can be viewed as a two degrees-of-freedom (2-DOF) mass-spring damper system whereby, one degree of freedom is the drive direction, and the second degree of freedom orthogonal to the first is the sense direction.

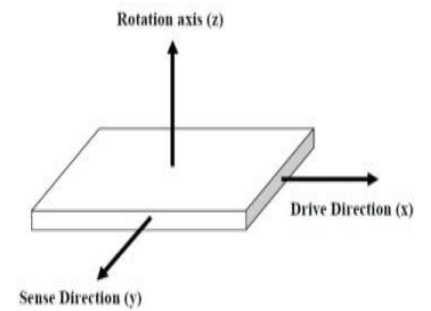

Fig 1.0: Generic model of a gyroscope

The coriolis acceleration is an apparent acceleration that arises in the rotation reference frame and it is proportional to the rate of rotation. The coriolis force couples the sense and drive directions of the gyroscope (figure 1.1). For understanding the dynamics and principle of operation of a gyroscope, the equations of motions of a simple mass-spring damper system (figure 1.1) has been derived [4]. 


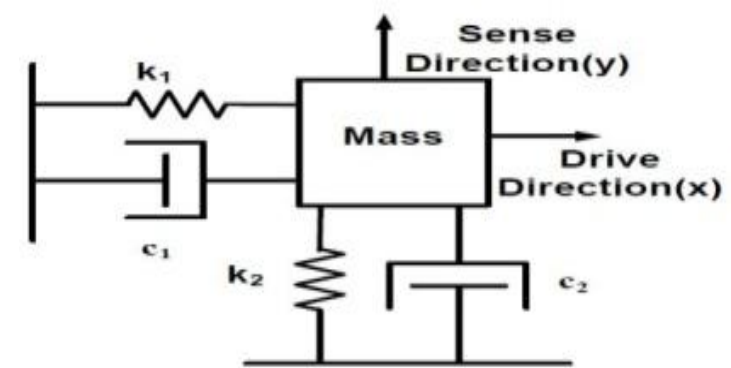

Fig 1.1: Two degrees-of-freedom (2-DOF) mass-spring damper system

We can write mathematical model equation.

$$
\sum \mathrm{F}=m \frac{\partial^{2} y}{\partial t^{2}}+c \frac{\partial y}{\partial t}+k y(1)
$$

Where,

$\mathrm{F}=$ actuation force on the drive direction

$\mathrm{m}=$ mass of proof body

$\mathrm{c}=$ damping coefficient

$\mathrm{k}=$ spring constant

Equation (1) holds in the inertial frame, where angular rotation is zero. For observing the actual vibration of the body in the rotating frame, Coriolis force induced due to rotation must be taken into consideration[5]. The acceleration experienced by a moving body in a rotating frame can be derived starting with the following definitions of figure 1.2

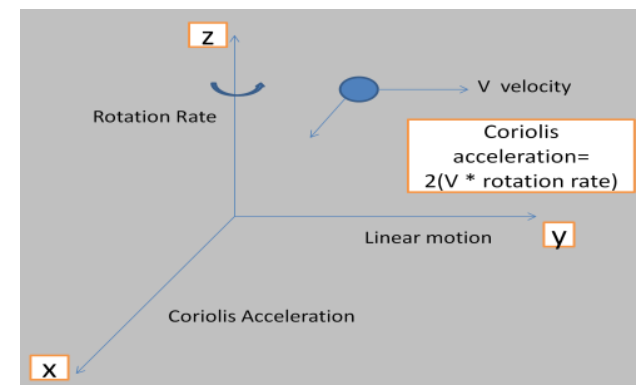

Fig. 1.2: Time derivative of a vector in a rotating frame

When the mass is driven along the $y$-direction by an external force $F(t)=F_{0} \sin \omega t$ and it is subjected to a constant angular rate $(\Omega)$, a Coriolis force $F^{*}=\left(2 \mathrm{~m} \Omega \frac{\partial \mathrm{y}}{\partial \mathrm{t}}\right)$ is inducedin then $\mathrm{x}$-direction. In this case, the equation ofmotion is

$$
\mathrm{m} \frac{\partial^{2} y}{\partial t^{2}}+k_{1} \frac{\partial y}{\partial t}+c_{1} y=F_{0} \sin \omega t(2)
$$

$\mathrm{m} \frac{\partial^{2} x}{\partial t^{2}}+k_{2} \frac{\partial x}{\partial t}+c_{2} x=2 \mathrm{~m} \Omega \frac{\partial \mathrm{y}}{\partial \mathrm{t}}(3)$

\section{Mechanical Design of a MEMS Gyroscope}

Most conventional micro mechanical gyroscopes developed are resonant sensors, they are basically resonators. These types of resonating sensors are having high bias stability, sensitivity, amplifications and immune to environmental interference. In this work the gyroscope has been made from a lame-mode resonator.

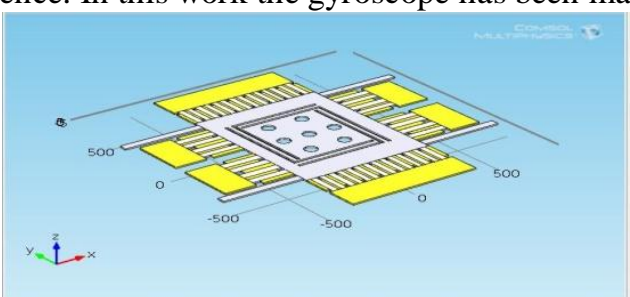

Fig. 1.4: Structure of MEMS Based Gyroscope

A micro-machined vibrating rate gyroscope shown in Fig. 1.4, in which the mass is supported by four anchors. The oscillator is $415 \mathrm{~mm} \times 535 \mathrm{~mm} \times 10 \mathrm{~mm}$ in size. It is driven by means of comb electrodes, and there are 
fourother comb electrodes to tune the frequency [1], [2]. When AC and DC voltages are applied to the comb electrodes in driving direction, the mass oscillates along the $y$-direction drive mode. An externally induced rotation about the z-axis, angular rate $(\Omega)$ produces a deflection in the $\mathrm{x}$-direction due to the Coriolis force. This deflection of proof mass has been detected as a change in the capacitance between the mass and the detection electrode. If the frequencyresponse mismatch $(\Delta f)$ between the drive and sense modes is small, the detection mode enhances the deflection.

Following materials and properties has been used for:

For Proof mass and anchors: Single Crystal Silicon

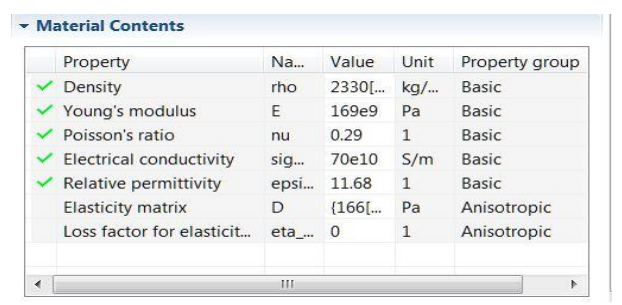

For Comb Electrodes: Gold

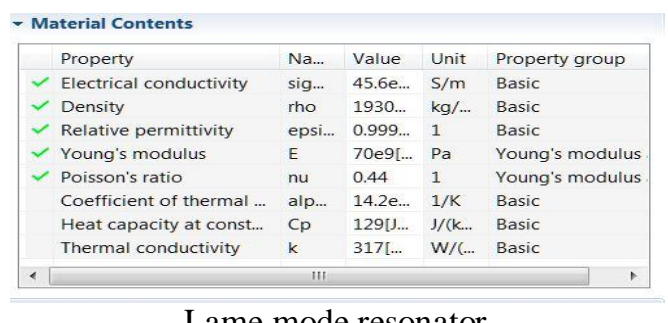

Lame mode resonator

The architecture of MEMS based gyroscope is a combination of a comb drive-mode oscillator thatinduces and sustains a constant linear or angular momentum, which has been coupled to a sense-modecoriolis accelerometer that detects the Coriolis force induced due to thecombination of the drive actuation and an angular rate input in the respective axis.In this project, the structure of the lame-mode resonator is a square plate (figure 1.4). Theplate is supported by 4 anchors. Two electrodes are used for exciting the resonator, four tosense the resonator motion [2]. The primary mode of vibration is a Lame-mode, in which theedges of the square plate bend in anti-phase, so preserving the volume of the plate Figure 1.4.

\subsection{Resonator theory}

Resonating systems are executed by equations of motion with one degree of freedom. Theresonator can be represented as a one dimensional mechanical damper system (figure 1.5). Wecan derive the equations as specified in equation (1) for governing the motion of the one dimensional damper system

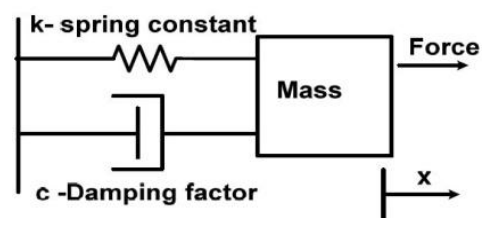

Fig 1.5: One degrees-of-freedom (1-DOF) mass-spring damper system

\section{MEMS Based Gyroscope Simulation}

For this project, simulation has been carried out with COMSOL Multiphysics 4.3 to analyze the performance and optimize the design of the MEMS based Gyroscope. The Eigen frequency analysis of drive \& sense mode and application of force over the proof mass has been described.

\subsection{Eigen Frequency analysis}

Eigen Frequency is one of the frequency at which an oscillatory system can vibrate. The main reason for this analysis is to know the different eigen frequencies of the rectangular plate. For each and different 
eigenfrequencies, proof mass of the plate vibrates and give different deflection. From the eigen frequency analysis, maximum deflection of proof mass can be shown.

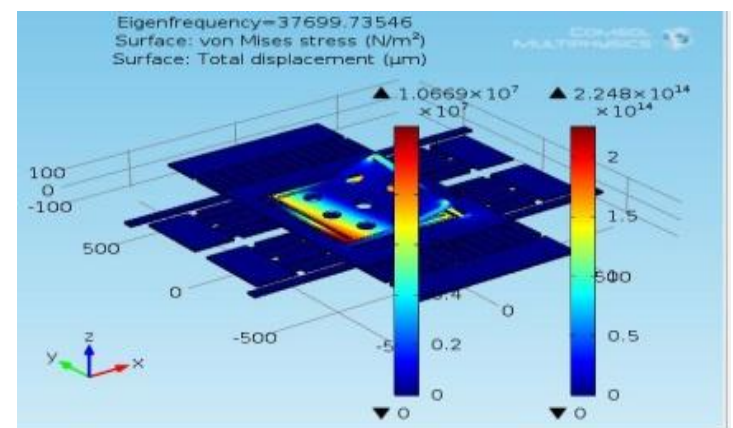

Fig. 1.6: Deflection of the proof mass at $21906.47525 \mathrm{~Hz}$.

Table 1.0: Deflection of proof mass for different Eigen frequencies.

\begin{tabular}{|l|l|l|l|l|l|l|}
\hline $\begin{array}{l}\text { Eigen } \\
\text { freque } \\
\text { ncy(in } \\
\text { Hz) }\end{array}$ & $\begin{array}{l}2190 \\
6.475\end{array}$ & $\begin{array}{l}37699 \\
.7354\end{array}$ & $\begin{array}{l}38374 . \\
6\end{array}$ & $\begin{array}{l}1.854 \\
415 \mathrm{e}\end{array}$ & $\begin{array}{l}2.494 \\
785 \mathrm{e} \\
5\end{array}$ & $\begin{array}{l}2.515 \\
951 \mathrm{e} \\
5\end{array}$ \\
\hline $\begin{array}{l}\text { Deflec } \\
\text { tion } \\
\text { (in } \mu \mathrm{m})\end{array}$ & $\begin{array}{l}5.551 \\
9\end{array}$ & $\begin{array}{l}1.066 \\
9\end{array}$ & 1.0795 & $\begin{array}{l}1.195 \\
6\end{array}$ & 1.997 & $\begin{array}{l}2.472 \\
2\end{array}$ \\
\hline
\end{tabular}

Frequency response of rectangular proof mass of the lame mode has been shown in graph $1.0 \& 1.1$. This has been done by giving the force in respective direction of the drive and sense mode of the proof mass.

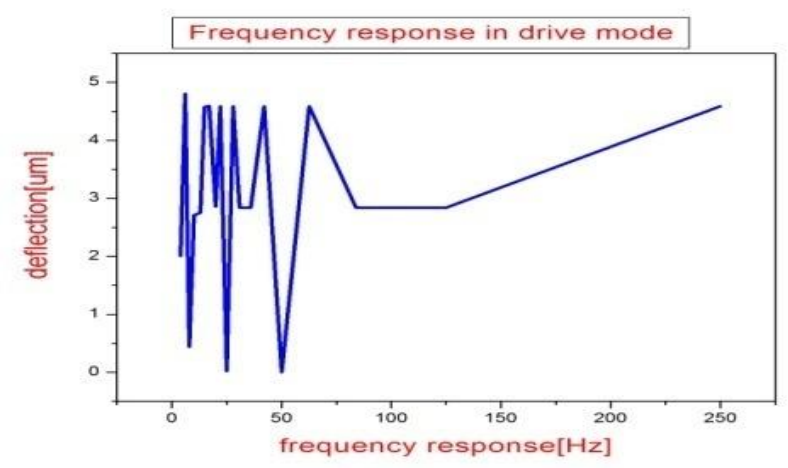

Fig. 1.7: Frequency response plot of the Drive Mode of the geometry

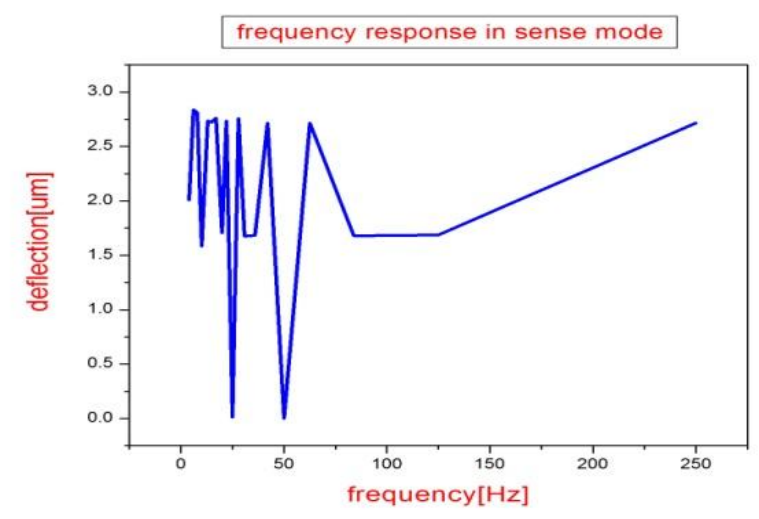

Fig 1.8: Frequency response plot of sense mode of the geometry.

The above two graphs has been shown of frequency response for drive mode (graph 1.0) and for sense mode (graph 1.1). The Frequency response of these two modes must be matched closely in order to enhance the performance of the MEMS based gyroscope. In order to match the drive mode and sense mode of gyroscope, the width of the anchors of the rectangular plate has been varied by using a COMSOL script to match the two 
resonance frequencies. Below graph 1.2 has been shown for resonance frequency versus thickness of the anchors, which is drawn by giving no boundary load and voltage to the geometry during simulation.

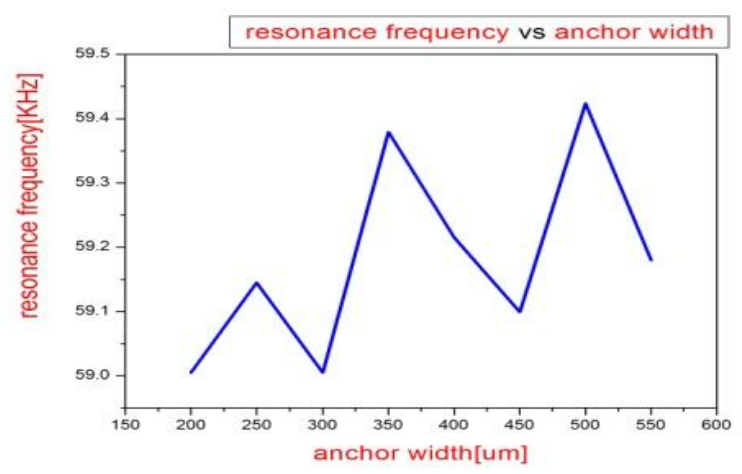

Fig 1.9: A plot of resonance frequency VersusThickness of the anchors

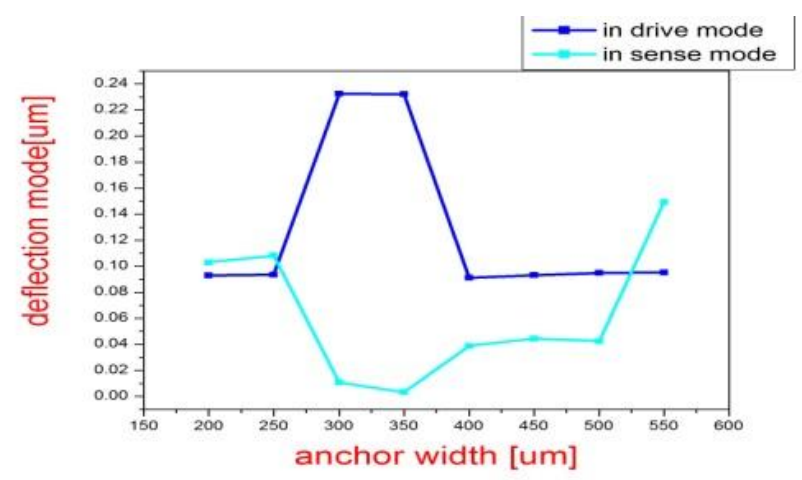

Fig 1.10: A plot deflection of geometry versus thickness of the anchors at resonance frequency $10.0167 \mathrm{~Hz}$.

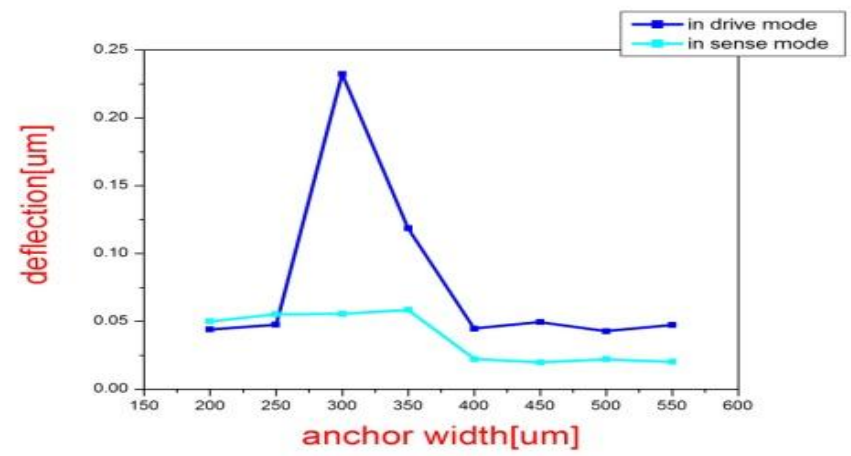

Fig 1.11: A plot deflection of geometry versus thickness of the anchors at resonance frequency $12.5133 \mathrm{~Hz}$

This figure 1.10 has been obtained from COMSOL multiphysics. From the plot, it has been deduced that,thickness of $525 \mu \mathrm{m}$ and $260 \mu \mathrm{mof}$ anchors, made the drive and sense mode matched.

\section{Results and Discussion}

The simulated result has been shown in this paper. The Eigen frequency analysis has been done in which proof mass of the MEMS based gyroscope has deflected for each Eigen frequency through which a change in capacitance can be measured. Maximum deflection of proof mass of $5.5519 \mu \mathrm{mhas}$ been noticed in this analysis. Also it has been proved that deflection of the proof mass has been changed as varying the width of anchors for both drive \& sense mode of the geometry. The figure 1.11 has been shown for voltage distribution over the proof mass when a electric potential has been applied to the geometry in Y- direction i.e. in direction of drive mode. 


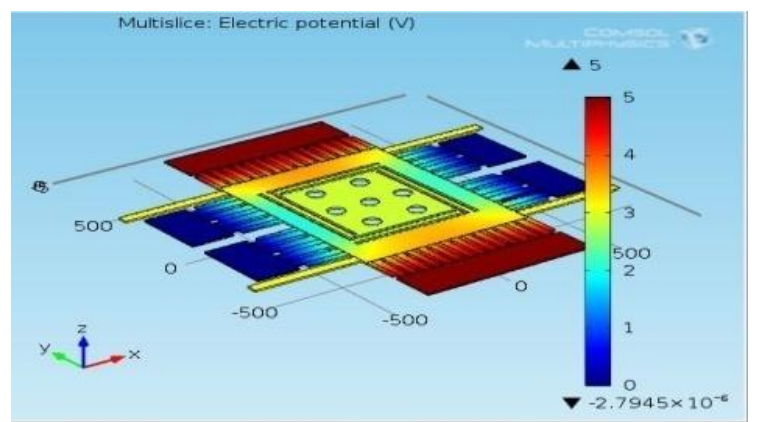

Fig. 1.12: Voltage Distribution over the proof mass.

\subsection{Capacitive detection}

The deflection of the proof mass has been measured in terms of change in capacitance by keeping a plate same as the size of the proof mass below the proof mass and distance between both plates has been covered by dielectric medium of $150 \mu \mathrm{m}$.

The sense electrode of the geometry is parallel to the rectangular proof mass. The parallel capacitance between the electrode and the proof mass is

$$
\mathrm{C}=\frac{\varepsilon_{0} A}{d}(4)
$$

Where, $\varepsilon_{0}=$ permittivity of the free space,

$\mathrm{A}=$ area between the sense electrode and the

Rectangular plate and

$d=$ gap between the electrode and the

Rectangular plate

When the device has been sensed by sense electrode in terms of change in capacitance between the sense electrode and device with deflection caused by coriolis force. The change in capacitance $\Delta C$ has been calculated as shown in equation:

$\Delta C=\frac{\varepsilon_{0} A}{d-\Delta d}-\frac{\varepsilon_{0} A}{d}(5)$

The change in capacitance is a nonlinear function of displacement in variable-gap capacitors. However, in the case that $\Delta d \leq d$, the change in capacitance varies linearly to the displacement.

$\Delta C=\frac{2 A \varepsilon_{0}}{d^{2}} \Delta d(6)$

In the presence of angular rotation, the device vibrates in sense mode. The asymmetrical shape of the sense mode results in the gap capacitances on the side sense electrode.

$C_{s+}=\frac{\varepsilon_{0} A}{d-\Delta d}(7)$

$$
C_{s-}=\frac{\varepsilon_{0} A}{d+\Delta d}(8)
$$

This change in capacitances has been measured and this measured value has been converted into voltage V1 \&V2 by connecting the two OP-AMPs on either side of the sense electrodes as shown in figure 1.12. 


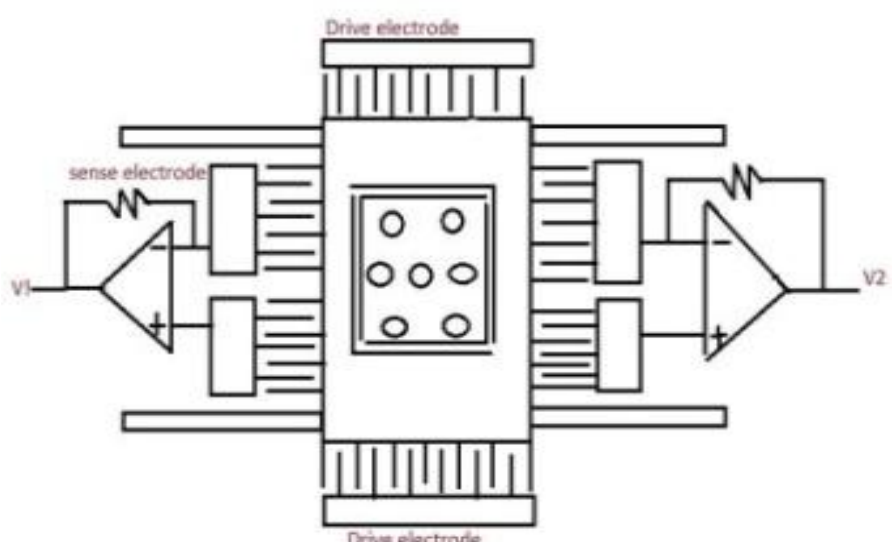

Fig. 1.13: Capacitive readout topology.

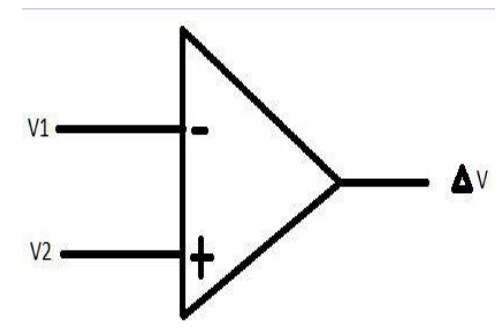

Fig. 1.14: Differential amplifier.

To detect the change in differential voltage of the pair of sensing electrodes of each side, differential amplifier has been used as shown in above figure 1.13.

In case of this project design with parameter:

$\mathrm{A}=$ width*height $=415 \times 535(\mu m)^{2}$

$\varepsilon_{0}=8.85 \times 10^{-12} \mathrm{~F} / \mathrm{m}$

$\mathrm{d}=150 \mu \mathrm{m}$

$$
\Delta d=5.5519 \mu m
$$

From equation (6) the change in capacitance has been calculated as $\Delta C=0.145 \mathrm{pF}$.

Hence, sensitivity of the capacitive readout of the given geometry has been assumed as change in capacitance value i.e. $0.145 p \mathrm{~F}$.

Now next step is to measure current due to sense mode oscillation. This has been done by imposing a constant DC bias voltage $V_{d c}$ over the sense electrode with capacitance

$$
C_{s}=C_{s e n}+\Delta C_{s} e^{j t \omega}(9)
$$

Current becomes

$$
i_{s}=\frac{\partial}{\partial t}\left[V_{d c} C_{s}(t)\right](10)
$$

$i_{s}=V_{d c} \omega_{s} \Delta C_{s} e^{j t \omega_{s}}(11)$

Hence expected current of this topology for given geometry with $\omega_{s}=2 \times \pi \times 21906.47525, \Delta C_{s}=0.145 p F V_{d c}=5 \mathrm{~V}$

$i_{s}=5 \times 2 \times \pi \times 21906.47525 \times 0.145 \times 10^{-12} \mathrm{~A}$

$i_{s}=0.9979 \times 10^{-7} \mathrm{~A}$

Now voltage has been calculated by using the value of feedback resistors. Value of the feedback resistors depends on the amplification.

$$
\begin{gathered}
\Delta V=K_{d} \times i_{s}(12) \\
\Delta V=0.5 M \Omega \times 0.9979 \times 10^{-7} \mathrm{~A}
\end{gathered}
$$




$$
\Delta V=49.895 \mathrm{mV}
$$

Furthermore, for improving the sensitivity, overall sensing area should be increased. However, the gap between the sensing electrode and the proof mass is the major factor that defines the capacitance sensitivity. This has been noticed from equation (6), in which change in capacitance $\Delta C$ varies inversely to the square of the gap (d) and linear to the overlap area.

\section{Conclusions}

In this paper a simulation of MEMS based gyroscope has been presented. The overview of this geometry has been given. MEMS based gyroscope has been designed to resonate in its fundamental mode and it has been exited by external comb drive electrode and sensed by comb sense electrode. This geometry enabled the matching of the drive mode and sense mode frequency by varying the width of the anchors of the geometry. The sensitivity of the given geometry for capacitive readout is $0.145 \mathrm{pF}$ and for the frequency of $21906.47525 \mathrm{~Hz}$, change in voltage has been calculated which is equal to $49.895 \mathrm{mV}$.

\section{References}

[1] DamrongritPiyabongkarn and Rajesh Rajamani“The Development of a MEMS Gyroscope for AbsoluteAngle Measurement”, Department of Mechanical Engineering University of Minnesota Minneapolis, MN 55455, Proceedings of the American ControlConference Anchorage, AK May 8-10, 2002

[2] P.J. Ngana, J.J. Koning “Design, modelling and simulation of a High Frequency MEMS Gyroscope in 1.5um SOI”, 2009(903-906), Proceedings of the Euro sensors XXIII conference.

[3] Yoichi Mochida, Masaya Tamura "A micro-machiened vibrating rate gyroscope with independent beams for the drive and detection modes" Yokohama Technical Center, Murata Manufacturing, 1-18-1, Hakusan, Midori-ku, Yokohama 226-0006, Japan , Sensors and Actuators 80 (2000) 170-178

[4] Jianli Li, Jiancheng Fang, Haifeng Dong, Ye Tao "Structure design and fabrication of a novel dual-mass Resonant output micromechanical gyroscope" MicrosystemsTechnol (2010) 16:543-552, DOI 10.1007/s00542-009-0998-8, 2010.

[5] Marcelo C. Algrain and James Quin "Accelerometer Based Line-of-Sight Stabilization Approach for Pointing andTracking Systems" Second IEEE Conference on Control Applications, September 13 - 16, 1993 Vancouver, B.C 